\title{
The relationship between X-ray and optical absorbers in active galactic nuclei
}

\author{
Gunnar W. Jaffarian` and C. Martin Gaskell† \\ Department of Astronomy and Astrophysics, University of California, Santa Cruz, CA 95064
}

\begin{abstract}
We present a large compilation of reddening estimates from broad-line Balmer decrements for active galactic nuclei (AGNs) with measured X-ray column densities. The median reddening is $E(B-V) \approx 0.77 \pm 0.10$ for type-1 to type-1.9 AGNs with reported column densities. This is notably higher than the median reddening of AGNs from the SDSS. We attribute this to the selection bias of the SDSS towards blue AGNs. For other AGNs we find evidence of a publication bias against reporting low column densities. We find a significant correlation between $N_{H}$ and $E(B-V)$ but with a large scatter of \pm 1 dex. On average the X-ray columns are consistent with those predicted from $E(B-V)$ for a solar neighbourhood dust-to-gas ratio. We argue that the large scatter of column densities and reddenings can be explained by X-ray column-density variability. For AGNs with detectable broad-line regions (BLRs) that have undergone significant changes of Seyfert type ("changing-look" AGNs) we do not find any statistically significant differences in $N_{H}$ or $E(B-V)$ compared to standard type-1 to type-1.9 AGNs. There is no evidence for any type-1 AGNs being Compton-thick. We also analyze type-2 AGNs and find no significant correlation between $N_{H}$ and narrow-line region reddening. We find no evidence for a previously claimed anti-correlation. The median column density of LINERs is $22.68 \pm 0.75$ compared to a column density of $22.90 \pm 0.28$ for type- 2 AGNs. We find the majority of low column density type-2 AGNs are LINERs, but $N_{H}$ is probably underestimated because of scattered light.
\end{abstract}

Key words: galaxies: active - galaxies: nuclei - galaxies: Seyfert - dust, extinction

\section{INTRODUCTION}

A large fraction of active galactic nuclei (AGNs) show Xray photoelectric absorption edges from which an equivalent hydrogen absorption column density, $N_{H}$, can be estimated. Reported column densities range from $<10^{20} \mathrm{~cm}^{-2}$ to $>10^{24} \mathrm{~cm}^{-2}$ (i.e., Compton thick). The nature and location of the absorbing material has been long debated. Optical spectrophotometry reveals that the line-emitting regions and continua of most AGNs are significantly reddened, with colour excesses, $E(B-V)$, of the order of 0.2 or much greater (see Gaskell 2017 for a review and references). Wherever there is dust there is also gas. Observations of the Milky Way give $N_{H}=5.8 \times 10^{21} E(B-V) \mathrm{cm}^{-2}$ (Bohlin et al. 1978). For this standard Milky Way dust-to-gas ratio, we would therefore expect that reddenings should range from $E(B-V) \approx 0.01$ (comparable to the reddenings at high Galac-

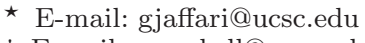

$\dagger$ E-mail: mgaskell@ucsc.edu tic latitudes in the solar neighbourhood) to $E(B-V) \gg 10$ for Compton-thick AGNs. Although there are many AGNs that are completely obscured in the optical (type-2 AGNs), there are also AGNs with very high column densities that do not show high reddenings in the optical (compare, for example the column densities given by Risaliti et al. 2000 and Shu et al. 2007 for Mrk 266 and NGC 3982 respectively with the $\mathrm{H} \alpha / \mathrm{H} \beta$ ratios given by Alam et al. 2015.) This situation has been commonly interpreted as being due to relatively dust-free X-ray absorbing gas. On the other hand, when there is significant reddening there must be gas present.

In the simplest unified models (see Antonucci 1993) Seyfert 2 galaxies are thermal AGNs seen at high inclination so that the surrounding dust blocks our view of the broadline region $(B L R)$ and accretion disk. There has therefore been much interest in the difference between X-ray absorbing properties of type-2 AGNs and type-1 AGNs. The naïve predictions are that type-2 AGNs should show high column densities and that type-1 AGNs should show low column densities. There is good evidence for this (see Goodrich et al. 
1994; Veilleux et al. 1997; Shi, et al. 2006; Burtscher, et al. 2016). Another prediction is that among type- 1 to type 1.9 AGNs, $N_{H}$ should be correlated with $E(B-V)$

By definition (Khachikian \& Weedman 1971), the optical spectra of type-2 AGNs only show lines from the narrow-line region (NLR). For the NLR the intensity ratio of $\mathrm{H} \alpha$ to $H \beta$, which we will simply refer to as the Balmer decrement, is expected to be the Case B value $(\approx 3$; see Osterbrock \& Ferland 2006). This is supported by observations of other line ratios (see Gaskell 1984 and Wysota \& Gaskell 1988). The reddening of the NLR can therefore readily be estimated from the observed Balmer decrement.

The studies of Dong et al. (2008) and Gaskell (2017) show that the intrinsic Balmer decrements of the higher density broad-line region (BLR) gas also have a Case B ratio and hence the reddening of the BLR can also be estimated from the Balmer decrement. Gaskell (2017) gets an intrinsic $\mathrm{H} \alpha / \mathrm{H} \beta$ ratio of $2.7^{1,2}$. Malizia et al. (1997) studied the relationship between $N_{H}$ and Balmer decrement for AGNs of all types (BLR decrements for types $1-1.9$ and NLR decrements for type 2). Contrary to the naïve prediction, they did not find significant correlations between the NLR or BLR Balmer decrements and $N_{H}$. Guainazzi et al. (2001) studied the relationship between $N_{H}$ and the NLR Balmer decrement in just type-2 AGNs. Contrary to predictions, they surprisingly report an anti-correlation between $N_{H}$ and NLR Balmer decrement (i.e., steeper Balmer decrements corresponding to lower column densities). A physical cause of this is hard to understand.

In this paper we therefore present a compendium of column densities and Balmer decrements for a substantially larger sample of type-1 to type-1.9 AGNs and discuss the relationship between reddening and column density and its significance. We also re-examine the relationship between column density and reddening for type-2 AGNs.

\section{DATA}

In Table 1 we present a compendium of AGN hydrogen column densities, Balmer decrements, and classifications taken from the literature. The criterion for inclusion was the availability of both an estimate of the hydrogen column density (including upper limits) from X-ray observations and a Balmer decrement. The data are necessarily inhomogeneous and subject to publication bias. Estimation of column densities is model-dependent (see, for example, Immler et al. 2003). Furthermore, as will be discussed below, column-density variability is very common (Reichert et al. 1985). We therefore do not quote errors for the column density even when they are estimated in the original sources. Malizia et al. (1997) found column-density variability in $70 \%$ of the sources they could analyze. Where there were multiple values of the column density available we simply give a geometric average weighted by the number of observations (but not by any error bars). Table 1 also gives BLR Balmer decrements for type 1 to $1.9 \mathrm{AGNs}$ and NLR Balmer decrements for type-2 AGNs and LINERS. For these estimates we favored references with the highest quality spectra. The majority of studies have not subtracted out host-galaxy starlight from spectra before calculating Balmer decrements. This results in a systematic overestimate of the Balmer decrement when $\mathrm{H} \beta$ emission is weak, especially when there is a young stellar population present with strong Balmer absorption lines. This does not affect our analysis.

In column (7) we give reddening estimates for the Balmer decrement. We have calculated $E(B-V)$ from broadline Balmer decrements using

$$
E(B-V)=2.5 \times 1.3 \times \log _{10}\left(\frac{\mathrm{H} \alpha / \mathrm{H} \beta}{2.7}\right),
$$

where the intrinsic $\mathrm{H} \alpha / \mathrm{H} \beta$ ratio for the BLR has been taken to be 2.7 following Gaskell (2017). The intrinsic $\mathrm{H} \alpha / \mathrm{H} \beta$ ratio has been taken to be 3.1 for the NLR. The ratio of selective extinction between $\mathrm{H} \alpha$ and $\mathrm{H} \beta$ compared to $E(B-V)$ has been taken to be 1.3 using the Milky Way reddening curve of Weingartner \& Draine (2001).

Table 1 contains 89 type- 1 to type- 1.9 AGNs, and 35 type-2 AGNs or LINERS (indicated by a "3" for their type), with their Balmer decrements and X-ray column densities. References to the sources of the measurements are given in the table. The organization of the table is as follows. AGNs are grouped by optical classification in order of increasing optical type. "Changing-look" AGNs, whose optical spectra have changed enough to change the Seyfert classification are placed at the end, followed by AGNs with only upper or lower limits on the hydrogen column density. These things are noted in column (8). All classifications for the galaxies come from the NASA/IPAC Extragalactic Database (NED). When there have been multiple type claims for the galaxies, we chose the most recent classification from NED's references. In some cases, if a claim that an object was type- 2 seemed unreasonable because of obvious broad Balmer lines in the spectra, we used an older classification that was more consistent with a non-type-2 classification.

\footnotetext{
1 The Case B ratio for the BLR is expected to be higher than for the NLR because the density is higher. For the same ionization parameter, NLR gas has a lower temperature, and hence a higher Case B ratio, because collisional-line cooling results in a lower temperature. See Osterbrock \& Ferland (2006).

2 Gaskell (2017) explains that the slightly higher $\mathrm{H} \alpha / \mathrm{H} \beta$ Dong et al. (2008) find for radio-loud AGNs and AGNs with double-peaked Balmer lines is a consequence of the higher reddening of AGNs seen at higher inclinations, rather than fundamentally different physical conditions in these AGNs
} 
Table 1. Column densities and reddenings

\begin{tabular}{|c|c|c|c|c|c|c|c|}
\hline (1) Object Name & (2) Class & (3) $\mathrm{H} \alpha / \mathrm{H} \beta$ & (4) $\mathrm{H} \alpha / \mathrm{H} \beta \operatorname{Ref}$ & (5) $\log \left(N_{\boldsymbol{H}}\right)$ & (6) $N_{H} \operatorname{Ref}$ & (7) $\mathrm{E}(\mathrm{B}-\mathrm{V})$ & (8) Notes \\
\hline 3C 111 & 1 & 15.00 & $44^{*}$ & 22.80 & $10,11,73(2)$ & 2.42 & \\
\hline $3 \mathrm{C} 120$ & 1 & 6.46 & 1,2 & 21.39 & 10,76 & 1.23 & \\
\hline $3 \mathrm{C} 445$ & 1 & 9.50 & 3 & 23.16 & $1,12,76$ & 1.78 & \\
\hline ESO 141-G055 & 1 & 4.00 & 8,9 & 21.66 & $1,76-$ & 0.55 & \\
\hline Fairall 51 & 1 & 3.32 & 10,9 & 21.90 & $13,76-$ & 0.29 & \\
\hline H 1846-786 & 1 & 2.40 & $41^{*}$ & 22.80 & 1 & -0.17 & \\
\hline I Zw 1 & 1 & 4.66 & 1,2 & 20.81 & 2 & 0.77 & \\
\hline IC 5063 & 1 & 5.53 & 11 & 23.48 & 12,76 & 1.01 & \\
\hline Mrk 1044 & 1 & 2.40 & $41^{*}$ & 20.62 & 3 & -0.17 & \\
\hline Mrk 110 & 1 & 4.22 & $1,2,7^{*}$ & 20.17 & $3,76-$ & 0.63 & \\
\hline Mrk 1239 & 1 & 3.50 & $41^{*}$ & 20.93 & 3 & 0.37 & \\
\hline Mrk 1310 & 1 & 3.97 & $7^{*}$ & 20.48 & 3 & 0.55 & \\
\hline Mrk 142 & 1 & 2.90 & $1,2,7^{*}$ & 20.40 & 3 & 0.10 & \\
\hline Mrk 205 & 1 & 4.28 & 13,2 & 20.35 & $3,76-$ & 0.65 & \\
\hline Mrk 279 & 1 & 4.90 & 12 & 22.90 & $5,73,76$ & 0.84 & \\
\hline Mrk 3 & 1 & 6.61 & 11 & 23.92 & $14,3,76$ & 1.26 & \\
\hline Mrk 304 & 1 & 2.86 & $1,2,14$ & 20.16 & 16 & 0.08 & \\
\hline Mrk 40 & 1 & 2.65 & $1,2,7^{*}$ & 20.36 & 3,76 & -0.02 & \\
\hline Mrk 42 & 1 & 3.63 & $5,2,7^{*}$ & 19.90 & 3 & 0.42 & \\
\hline Mrk 474 & 1 & 3.09 & 6,2 & 20.49 & 3 & 0.19 & \\
\hline Mrk 478 & 1 & 4.32 & 1,2 & 20.30 & 2 & 0.66 & \\
\hline Mrk 493 & 1 & 2.71 & $7^{*}$ & 20.47 & 3 & 0.00 & \\
\hline Mrk 5332 & 1 & 5.00 & 11 & 24.00 & 17 & 0.87 & \\
\hline Mrk 79 & 1 & 5.53 & 12 & 20.97 & $1,15,3,76-$ & 1.01 & \\
\hline Mrk 876 & 1 & 5.20 & 15,6 & 19.63 & 18 & 0.93 & \\
\hline NGC 2110 & 1 & 8.13 & 11 & 22.48 & $1,10,11,5,76$ & 1.56 & \\
\hline NGC 4051 & 1 & 3.50 & $11,6,2$ & 22.32 & $\begin{array}{l}11,22,23,24 \\
3,73(2), 76-\end{array}$ & 0.37 & \\
\hline NGC 4507 & 1 & 5.02 & 11 & 23.68 & $12,25,76$ & 0.88 & \\
\hline NGC 4593 & 1 & 2.61 & 17,18 & 21.23 & $1,5,76-$ & -0.05 & \\
\hline NGC 985 & 1 & 4.67 & 6 & 20.77 & 3,76 & 0.77 & \\
\hline PG $1001+054$ & 1 & 3.20 & $7^{*}$ & 19.37 & 30 & 0.24 & \\
\hline PG $1244+026$ & 1 & 2.95 & $7^{*}$ & 19.49 & 30 & 0.12 & \\
\hline PG $1448+273$ & 1 & 3.40 & $7^{*}$ & 19.64 & 31 & 0.33 & \\
\hline [HB89] $0241+622$ & 1.2 & 16.22 & 19 & 21.91 & 12,76 & 2.53 & \\
\hline Fairall 9 & 1.2 & 2.59 & 10,8 & 20.30 & $1,76-$ & -0.06 & \\
\hline IC $4329 A$ & 1.2 & 11.33 & 11,20 & 21.43 & $\begin{array}{l}1,10,11,35 \\
76\end{array}$ & 2.02 & \\
\hline Mrk 335 & 1.2 & 2.60 & 1,2 & 20.55 & 1,76 & -0.05 & \\
\hline Mrk 50 & 1.2 & 3.30 & $6,2,7^{*}$ & 19.90 & $5,76-$ & 0.29 & \\
\hline Mrk 705 & 1.2 & 4.40 & $7^{*}$ & 20.25 & $31,3,76-$ & 0.69 & \\
\hline NGC 4235 & 1.2 & 10.54 & $25,7^{*}$ & 21.29 & 3,76 & 1.92 & \\
\hline PG 1302-102 & 1.2 & 2.00 & $42^{*}$ & 19.43 & 29 & -0.42 & \\
\hline UGC 6728 & 1.2 & 7.00 & $45^{*}$ & 19.74 & $37,76-$ & 1.34 & \\
\hline MCG -2-58-22 & 1.5 & 5.80 & $7^{*}$ & 21.38 & $1,76-$ & 1.08 & \\
\hline MCG 8-11-11 & 1.5 & 4.28 & 12,26 & 21.17 & 1,76 & 0.65 & \\
\hline MR 2251-178 & 1.5 & 5.62 & 27 & 21.56 & $1,12,73,76-$ & 1.03 & \\
\hline Mrk 1152 & 1.5 & 3.20 & $41^{*}$ & 20.31 & $1,3,76-$ & 0.24 & \\
\hline Mrk 290 & 1.5 & 3.10 & $1,2,7^{*}$ & 21.67 & $1,3,76-$ & 0.20 & \\
\hline Mrk 509 & 1.5 & 2.69 & 1,2 & 20.81 & $1,76-$ & -0.01 & \\
\hline Mrk 817 & 1.5 & 4.00 & $12,23,24$ & 20.06 & $38,76-$ & 0.55 & \\
\hline Mrk 841 & 1.5 & 4.80 & $12,23,24$ & 20.20 & $5,76-$ & 0.81 & \\
\hline NGC 1275 & 1.5 & 10.71 & 29 & 21.80 & $22,39,76$ & 1.94 & \\
\hline NGC 1566 & 1.5 & 4.18 & 30,31 & 19.73 & $31,76-$ & 0.62 & \\
\hline Mrk 1218 & 1.8 & 9.04 & $7^{*}$ & 20.65 & 3 & 1.71 & \\
\hline Mrk 334 & 1.8 & 4.85 & 39 & 20.64 & 47 & 0.83 & \\
\hline Mrk 516 & 1.8 & 30.00 & 39 & 21.59 & 3 & 3.40 & \\
\hline Mrk 609 & 1.8 & 4.95 & $7^{*}$ & 20.77 & 3 & 0.86 & \\
\hline Mrk 744 & 1.8 & 5.47 & 39 & 22.07 & 3,76 & 1.00 & \\
\hline NGC 3660 & 1.8 & 7.50 & $41^{*}$ & 20.26 & 58 & 1.44 & \\
\hline NGC 4395 & 1.8 & 4.20 & $7^{*}$ & 21.51 & $11,5,76$ & 0.62 & \\
\hline Mrk 883 & 1.9 & 4.43 & $7^{*}$ & 21.15 & 3 & 0.70 & \\
\hline
\end{tabular}


Table 1. Continued

\begin{tabular}{|c|c|c|c|c|c|c|c|}
\hline (1) Object Name & (2) Class & (3) $\mathrm{H} \alpha / \mathrm{H} \beta$ & (4) $\mathrm{H} \alpha / \mathrm{H} \beta \operatorname{Ref}$ & (5) $\log \left(N_{H}\right)$ & (6) $N_{H} \operatorname{Ref}$ & (7) $\mathrm{E}(\mathrm{B}-\mathrm{V})$ & (8) Notes \\
\hline NGC 4138 & 1.9 & 6.50 & $46^{*}$ & 22.63 & 11,76 & 1.24 & \\
\hline NGC 4258 & 1.9 & 10.00 & $7^{* *}$ & 22.81 & $\begin{array}{l}49,50,11,3 \\
76\end{array}$ & 1.85 & \\
\hline NGC 4388 & 1.9 & 5.85 & $11,7^{*}$ & 23.12 & $51,11,5,3,76$ & 1.09 & \\
\hline NGC 4579 & 1.9 & 1.40 & $43^{*}$ & 19.30 & 11 & -0.93 & \\
\hline NGC 4594 & 1.9 & 20.00 & $7^{*}$ & 20.40 & 3 & 2.83 & \\
\hline NGC 5252 & 1.9 & 4.08 & $35,7^{*}$ & 22.45 & 9,76 & 0.58 & \\
\hline NGC 526A & 1.9 & 3.00 & 11 & 22.19 & $48(8), 76$ & 0.15 & \\
\hline NGC 5273 & 1.9 & 4.24 & $7^{*}$ & 20.95 & 11 & 0.64 & \\
\hline NGC 5506 & 1.9 & 7.22 & $11,7^{*}$ & 23.94 & $\begin{array}{l}1,10,11,52 \\
48,73,75,76\end{array}$ & 1.39 & \\
\hline NGC 7314 & 1.9 & 20.00 & 11 & 21.72 & $1,11,5,76$ & 2.83 & \\
\hline Cen A & 2 & 3.85 & 34 & 23.06 & 12,76 & 0.31 & \\
\hline Cygnus A & 2 & 8.50 & $42^{*}$ & 23.49 & 12,76 & 1.42 & \\
\hline ESO 103-G35 & 2 & 12.06 & 11 & 23.17 & $\begin{array}{l}26,1,12,73+, \\
76\end{array}$ & 1.92 & \\
\hline F01475-0740 & 2 & 5.72 & $41^{*}$ & 21.59 & 58 & 0.86 & \\
\hline IRAS 04575-7537 & 2 & 4.53 & 36 & 22.33 & 52,76 & 0.54 & \\
\hline IRAS 18325-5926 & 2 & 9.91 & 37 & 22.12 & 12,76 & 1.64 & \\
\hline MCG 5-23-16 & 2 & 7.24 & $11,7^{*}$ & 22.12 & $1,10,11,53$ & 1.20 & \\
\hline Mrk 270 & 2 & 3.78 & 39 & 23.18 & 56 & 0.28 & \\
\hline Mrk 348 & 2 & 6.02 & 11 & 23.10 & $26,53,76$ & 0.94 & \\
\hline Mrk $463 \mathrm{E}$ & 2 & 5.62 & 11 & 23.51 & 54 & 0.84 & \\
\hline Mrk 573 & 2 & 4.20 & 39 & 22.32 & 9,3 & 0.43 & \\
\hline Mrk 78 & 2 & 6.50 & 39 & 22.76 & 55 & 1.05 & \\
\hline NGC 1320 & 2 & 4.25 & $41^{*}$ & 23.60 & 19 & 0.45 & \\
\hline NGC 1667 & 2 & 9.74 & 16 & 24.66 & $57,58,14$ & 1.62 & \\
\hline NGC 1672 & 2 & 6.99 & 16 & 21.80 & 57 & 1.15 & \\
\hline NGC 1808 & 2 & 14.17 & 38 & 23.02 & 57,59 & 2.15 & \\
\hline NGC 3281 & 2 & 4.48 & $41^{*}$ & 23.63 & 5,76 & 0.52 & \\
\hline NGC 5643 & 2 & 5.58 & 16 & 22.92 & $12,63,77$ & 0.83 & \\
\hline NGC 5929 & 2 & 5.13 & $7^{*}$ & 20.71 & 3 & 0.71 & \\
\hline NGC 7172 & 2 & 6.50 & 34 & 22.89 & $26,1,5,76$ & 1.05 & \\
\hline NGC 7590 & 2 & 3.65 & $41^{*}$ & 20.96 & 58 & 0.23 & \\
\hline Mrk 266SW & 3 & 6.88 & $7^{*}$ & 20.61 & 3 & 1.12 & \\
\hline NGC 2655 & 3 & 4.70 & $41^{*}$ & 22.48 & 60 & 0.59 & \\
\hline NGC 3079 & 3 & 20.00 & $7+^{*}$ & 24.08 & $58,11,76$ & 2.63 & \\
\hline NGC 4278 & 3 & 3.55 & $43^{*}$ & 20.83 & 3 & 0.19 & \\
\hline NGC 5005 & 3 & 5.17 & $7^{*}$ & 22.88 & 58,3 & 0.72 & \\
\hline NGC 6240 & 3 & 20.50 & $46^{*}$ & 23.81 & $64,3,76$ & 2.67 & \\
\hline $3 \mathrm{C} 273$ & 1 & 3.05 & $42^{*}$ & 21.37 & $1,76-$ & 0.17 & changing look \\
\hline Ark 120 & 1 & 3.40 & 4 & 21.40 & $1,76-$ & 0.33 & changing look \\
\hline MCG -6-30-15 & 1.2 & 6.13 & $21,22,9,7^{*}$ & 21.96 & $1,11,73$ & 1.16 & changing look \\
\hline NGC 3227 & 1.5 & 5.43 & 11 & 22.27 & $\begin{array}{l}10,11,40,3 \\
73(3), 76\end{array}$ & 0.98 & changing look \\
\hline NGC 3783 & 1.5 & 3.37 & 11,30 & 21.78 & $\begin{array}{l}1,10,11,42, \\
43,24,73(2), \\
76\end{array}$ & 0.31 & changing look \\
\hline NGC $1365^{2}$ & 1.8 & 27.00 & 40 & 23.67 & $4,5,72,73(2)$ & 3.25 & changing look \\
\hline NGC 2992 & 2 & 7.08 & 39 & 21.71 & $7,8,5,3,74$ & 1.17 & changing look \\
\hline NGC 4941 & 2 & 7.50 & $41^{*}$ & 23.61 & 61,76 & 1.25 & changing look \\
\hline Mrk 590 & 1 & 4.19 & 1,6 & 20.54 & $1,2,3,71-$ & 0.62 & changing look \\
\hline NGC 7582 & 1 & 8.32 & 11 & 23.69 & $\begin{array}{l}26,1,26,27, \\
76\end{array}$ & 1.59 & changing look \\
\hline NGC 7469 & 1.2 & 5.42 & 12 & 21.06 & $1,5,3,76$ & 0.98 & changing look \\
\hline NGC 3516 & 1.5 & 3.47 & $11,1,2,32$ & 23.34 & $\begin{array}{l}11,41,5, \quad 70 \\
73,76-\end{array}$ & 0.35 & changing look \\
\hline NGC 5548 & 1.5 & 4.65 & $11,12,7^{*}$ & 21.41 & $\begin{array}{l}1, \quad 10, \\
45(2), 76\end{array}$ & 0.77 & changing look \\
\hline NGC 6814 & 1.5 & 3.67 & 6,2 & 22.01 & 1,76 & 0.43 & changing look \\
\hline Mrk 1018 & 1.9 & 6.82 & 39 & 20.34 & $3,76-$ & 1.31 & changing look \\
\hline NGC 4151 & 1.5 & 3.10 & 11 & 22.56 & $\begin{array}{l}26,1,10,41, \\
44,24,3,73+ \\
76\end{array}$ & 0.19 & changing look \\
\hline NGC $3982^{2}$ & 1.9 & 4.50 & $7^{*}$ & 24.00 & 9 & 0.72 & lower limit \\
\hline
\end{tabular}


Table 1. Continued

\begin{tabular}{|c|c|c|c|c|c|c|c|}
\hline (1) Object Name & (2) Class & (3) $\mathrm{H} \alpha / \mathrm{H} \beta$ & (4) $\mathrm{H} \alpha / \mathrm{H} \beta$ Ref & (5) $\log \left(N_{H}\right)$ & (6) $N_{H}$ Ref & (7) $\mathrm{E}(\mathrm{B}-\mathrm{V})$ & (8) Notes \\
\hline Mrk 266 & 2 & 4.55 & $7^{*}$ & 25.00 & 4 & 0.54 & lower limit \\
\hline Mrk 1040 & 1 & 6.39 & 6 & 21.56 & $1,12(3), 76$ & 1.21 & upper limit \\
\hline NGC 1068 & 1 & 7.38 & 16 & 24.22 & $\begin{array}{l}51,1,65,22, \\
66,11,67,3, \\
76+\end{array}$ & 1.42 & upper limit \\
\hline NGC 4639 & 1 & 1.809 & $7^{*}$ & 19.00 & 11 & -0.57 & upper limit \\
\hline Messier 81 & 1.8 & 7.60 & 34 & 21.56 & $22,68,11,3$ & 1.46 & upper limit \\
\hline NGC 5033 & 1.8 & 4.67 & $7^{*}$ & 19.94 & 11,3 & 0.77 & upper limit \\
\hline III Zw 2 & 2 & 3.50 & 1,2 & 21.97 & $1,12,76$ & 0.17 & upper limit \\
\hline NGC 1058 & 2 & 5.10 & $47^{*}$ & 20.78 & 11 & 0.70 & upper limit \\
\hline NGC 3185 & 2 & 6.28 & $7^{*}$ & 20.30 & 11 & 1.00 & upper limit \\
\hline NGC 5194 & 2 & 2.05 & $47^{*}$ & 24.00 & 69 & -0.58 & upper limit \\
\hline
\end{tabular}

Table 1. References

H $\alpha / \mathrm{H} \beta$ References: (1) Osterbrock 1977; (2) de Bruyn \& Sargent 1978; (3) Osterbrock et al. 1976; (4) Kollatschny et al. 1981; (5) Phillips 1978; (6) Rudy 1984; (7) Alam et al. 2015; (8) Ward et al. 1978; (9) Glass et al. 1982; (10) Hawley \& Phillips 1978; (11) Mulchaey et al. 1994; (12) Cohen 1983; (13) Neugebauer et al. 1979; (14) Kunth \& Sargent 1979; (15) Grandi 1981; (16) Storchi-Bergmann et al. 1995; (17) MacAlpine et al. 1979; (18) Ward et al. 1982; (19) Margon \& Kwitter 1978; (20) Wilson \& Penston 1979; (21) Morris \& Ward 1988; (22) Pineda et al. 1980; (23) Markarian 1977; (24) Denisyuk \& Lipovetskii 1977; (25) Abell et al. 1978; (26) Lacy et al. 1982; (27) Canizares et al. 1978; (28) Rieke 1978; (29) Phillips et al. 1983; (30) Osmer et al. 1974; (31) Glass 1981; (32) McAlary et al. 1979; (33) Tohline \& Osterbrock 1976; (34) Rebecchi et al. 1992; (35) Osterbrock \& Martel 1993; (36) de Grijp et al. 1992; (37) White et al. 1994; (38) Véron-Cetty \& Véron 1986; (39) Dahari \& De Robertis 1988; (40) Edmunds \& Pagel 1982; (41) Jones et al. 2009; (42) Torrealba et al. 2012; (43) Ho et al. 1995; (44) Buttiglione et al. 2009; (45) Falco et al. 1999; (46) Moustakas \& Kennicut 2006; (47) Rosales-Ortega et al. 2010; (*) Calculated from spectra from the reference; $\left({ }^{*}\right)$ Estimated from spectra from the reference
$\mathrm{N}_{\boldsymbol{H}}$ References $^{3}$ (1) Turner \& Pounds 1989; (2) Boller et al. 1996; (3) Pfefferkorn et al. 2001; (4) Risaliti et al. 2000; (5) Winter et al. 2009; (6) Guanazzi et al. 2002; (7) Weaver et al. 1996; (8) Mushotzky 1982; (9) Shu et al. 2007; (10) Weaver et al. 1995; (11) Nandra \& Pounds 1994; (12) Malizia et al. 1997; (13) Jiménez-Bailón et al. 2008; (14) Bianchi et al. 2005a; (15) Tueller et al. 2008; (16) Kartje et al. 1997; (17) Bianchi et al. 2005b; (18) Schartel et al. 1996; (19) BalokoviÄĞ et al. 2014; (20) Eracleous et al. 2010; (21) Ruschel-Dutra et al. 2014; (22) Brinkmann \& Siebert 1994; (23) Mihara et al. 1994; (24) Crenshaw \& Kraemer 2012; (25) Matt et al. 2004; (26) Warwick et al. 1993; (27) Rivers et al. 2015; (28) Nardini et al. 2015; (29) Rachen et al. 1996; (30) Wang et al. 1996; (31) Walter \& Fink 1993; (32) Saez et al. 2012; (33) Marinucci et al. 2014; (34) Różańska et al. 2004; (35) Madejski et al. 1995; (36) Miniutti et al. 2010; (37) Winter et al. 2008; (38) Winter et al. 2010; (39) Rhee et al. 1994; (40) Markowitz et al. 2009; (41) Morse et al. 1995; (42) Turner et al. 1993; (43) George et al. 1995; (44) Weaver er al. 1994; (45) Mehdipour et al. 2015; (46) Ursini et al. 2015; (47) Prieto et al. 2002; (48) Risaliti et al. 2002; (49) Pietsch et al. 1994; (50) Makishima et al. 1994; (51) Cappi et al. 2006; (52) Smith \& Done 1996; (53) Mulchaey et al. 1993; (54) Imanishi \& Terashima 2004; (55) Gilli et al. 2010; (56) Guainazzi et al. 2005; (57) Awaki \& Koyama 1993; (58) Ying et al. 2012; (59) Junkes et al. 1995; (60) González-Martín 2008; (61) Vasudevan et al. 2013; (62) Puccetti et al. 2014; (63) Guainazzi et al. 2004; (64) Puccetti et al. 2016; (65) Marshall et al. 1993; (66) Ueno et al. 1994; (67) Bauer et al. 2015; (68) Petre et al. 1993; (69) Fukazawa et al. 2001; (70) Turner et al. 2011; (71) Rivers et al. 2012; (72) Risaliti et al. 2005; (73) Gofford et al. 2013; (74) Shu et al. 2010; (75) Sun et al. 2017; (76) Ricci et al. 2017; (77) Matt et al. 2013; (78) Wang et al. 2010; (79) Zhang et al. 2006; (80) Yamada et al. 2018; (81) Barcons et al. 2003; (82) Piconcelli et al. 2004; (83) Gallo et al. 2006

2 See text

${ }^{3}$ It is left unclear as to whether Galactic column densities are subtracted from the column density in most references, but the usual small adjustment for Galactic column density does not affect our analysis 
Table 2. Median column densities and reddenings for different AGN types.

\begin{tabular}{ccc}
\hline $\begin{array}{c}\text { AGN type } \\
(1)\end{array}$ & $\begin{array}{c}\text { Median } \log \left(N_{\boldsymbol{H}}\right)\left(\mathrm{cm}^{-2}\right) \\
(2)\end{array}$ & $\begin{array}{c}\text { Median } E(B-V) \\
(3)\end{array}$ \\
\hline 1 & $20.93 \pm 0.24$ & $0.63 \pm 0.12$ \\
1.5 & $21.49 \pm 0.31$ & $0.59 \pm 0.15$ \\
1.8 & $21.14 \pm 0.43$ & $1.22 \pm 0.40$ \\
1.9 & $22.19 \pm 0.50$ & $1.09 \pm 0.35$ \\
All $1-1.9$ & $21.26 \pm 0.18$ & $0.77 \pm 0.10$ \\
2 & & \\
LINER & $22.90 \pm 0.28$ & $0.85 \pm 0.14$ \\
\hline
\end{tabular}

\section{ANALYSIS}

Table 2 gives the median column densities with errors in the medians and median reddenings for the various optical subclasses. It should be remembered that in Tables 1 and 2 the reddenings of the type- 1 to type-1.9 AGNs refer to the BLR, whilst the reddenings of the type-2 AGNs and LINERs refer to the NLR.

\section{$3.1 \quad$ Type-1s and intermediates}

The median reddenings of the BLRs of the type-1.8 and 1.9 AGNs in Table 2 are not unusual (see Heard \& Gaskell 2016). However, for type- 1 and 1.5 AGNs the median reddenings are approximately three times the median reddening of type-1 AGNs in the SDSS calculated assuming the same intrinsic BLR Balmer decrement (Gaskell 2017). This is not surprising since SDSS AGNs are heavily biased towards bluer AGNs because of the SDSS colour selection. The type- 1 to type-1.5 AGNs in Table 2 also have reddenings some 50\% higher than the reddenings Gaskell (2017) found for the Seyfert 1s of Osterbrock (1977). The higher median $E(B-V)$ in Table 2 points to reported column densities being biased against low X-ray columns and reddenings. To test this we looked at the reddenings of the Osterbrock (1977) AGNs with and without reported X-ray columns. For those without reported $\mathrm{X}$-ray columns the median reddening is $E(B-V)=0.31 \pm 0.10$ whilst for those with reported X-ray columns $E(B-V)=0.63 \pm 0.12$. This supports the conclusion that there is a bias towards reporting X-rays columns for AGN with higher column densities and higher reddenings.

Figure 1 shows the relationship between $E(B-V)$ and $N_{H}$ for type-1 to type-1.9 Seyferts. This is a sample size about three times larger than that of the Malizia et al. (1997) sample. It can be seen that there is a modest correlation between column density and reddening. The Spearman rank correlation coefficient $r=0.43$, giving a single-tailed probability $p=1.43 * 10^{-5}$, for the null hypothesis of no correlation. If we assume, following Malizia et al. (1997), that the scatter is mostly in $N_{H}$ and perform a regression of $\log N_{H}$ on $\log E(B-V)$ we get a slope that is very similar to the linear relationship of Bohlin et al. (1978). If we assume equal errors in $N_{H}$ and $\log E(B-V)$ and calculate a linear OLS bisector fit (Isobe et al. 1990) it can be seen in Figure 1 that we get a steeper slope.

As an illustration of the effect of publication bias against reporting non-detections of $N_{H}$ we show in the Figure 2

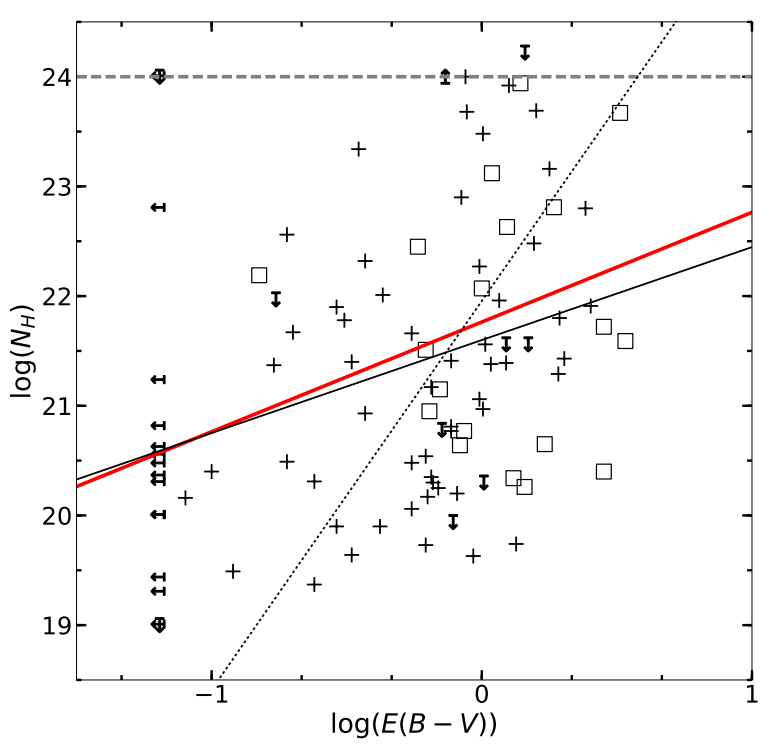

Figure 1. X-ray column densities versus $\log E(B-V)$ for 89 type1 and intermediate AGN. Type-1 and type-1.5 AGNS are shown as black crosses and type-1.8 and type-1.9 AGNs are shown as hollow squares. For negative reddenings in Table $1, \log E(B-V)$ has been set to -1.2 . The thick red line indicates the expected relationship between column density and reddening from Bohlin et al. (1978) and the dashed horizontal line indicates where an AGN becomes Compton-thick. The solid black line is a linear regression of $\log N_{H}$ on $\log E(B-V)$ (i.e., assuming that errors in $\log N_{H}$ dominate) and the dotted line is a linear OLS bisector fit (Isobe et al. 1990) assuming roughly equal errors in each axis.

the effects of assuming that the 18 AGNs from Osterbrock (1977) without published column densities had $\log N_{H}=20$ (i.e., towards the lower end of the range in Figure 1). This raises the Spearman rank correlation coefficient to $r=0.47$ lowering the single-tailed probability of the null hypothesis being correct to $p=1.8 * 10^{-7}$. As can be seen by comparing Figures 1 and 2, the effect on the slope of the linear regression of $\log N_{H}$ on $\log E(B-V)$ and the offset of this from the Bohlin et al. (1978) relationship is small.

\subsection{Comparing type-1 to type-1.9 AGNs, type-2 AGNs, and LINERS}

It has long been known that type-2 AGNs have higher column densities than type-1 AGNs (e.g., Malizia et al. 1997) and the larger sample presented here confirms this. A t-test comparing the column densities for type- 1 and intermediate AGNs to type- 2 AGNs in Table 1 gives a probability of only $p=1.25 \times 10^{-6}$ (single-tailed) for the $N_{H}$ distributions being the same.

In Table 2 the median $\log N_{H}$ for both the Seyfert $2 \mathrm{~s}$ and the LINERS is an order of magnitude larger than for the type-1 to type-1.9 AGNs. Both the X-ray column densities and the median reddenings of the NLRs of the two classes appear to be similar. The similar reddenings implies 


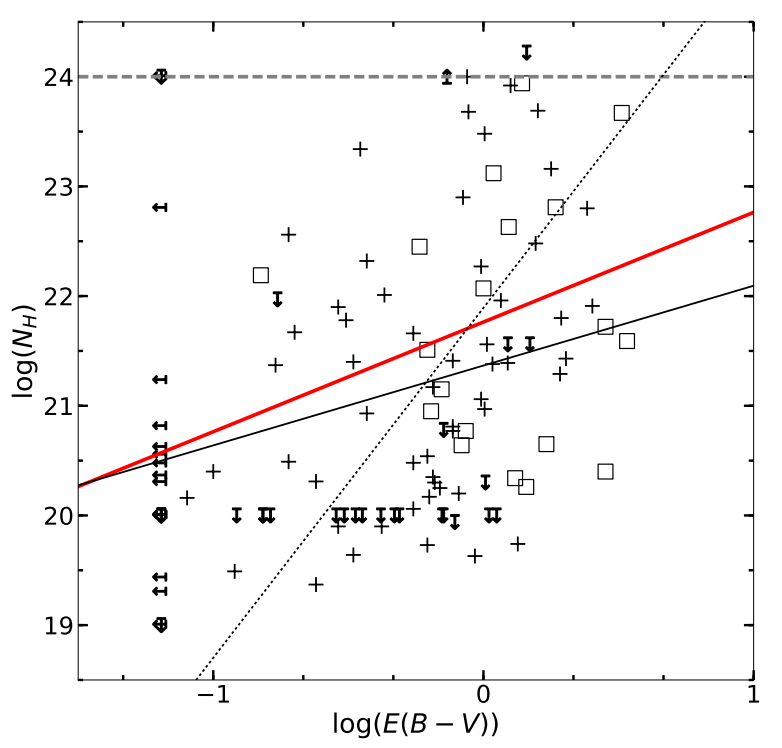

Figure 2. Same as Figure 1 except that we have included as upper limits in $\log N_{\boldsymbol{H}}$ the 18 AGNs from Osterbrock (1977) that do not yet have reported hydrogen column densities. We have assumed upper limits of $\log N_{H}=20$. Note there is only a slight change in the regression lines. This indicates that publication bias does not have a strong effect on the correlations.

that the amount of dust in or around the regions producing the narrow emission lines is similar in the two classes of object. There is no evidence from the information considered here that the type-2 AGNs and LINERS are fundamentally different (see Ho 2008 and Antonucci 2012 for extensive discussion).

\subsection{The relationship between X-ray column density and NLR reddening for type-2 AGNs}

The NLR is considerably more extended than the BLR and X-ray-emitting regions of an AGN. Comparison of NLR and BLR reddenings (see Figure 3 of Heard \& Gaskell 2016) shows that the BLR reddening is generally at least the same as the NLR reddening, but frequently greater. Heard \& Gaskell (2016) demonstrate that the relationship between NLR and BLR reddenings and relationships between other quantities (equivalent widths and broad/narrow line ratios) are consistent with the bulk of the dust blocking the inner regions of an AGN being located between the NLR and BLR, but with more distant dust, either associated with the NLR or in the host galaxy, also contributing.

Figure 3 shows the relationship between $N_{H}$ and the reddening of the NLR for the type-2 AGNs and LINERs. As can be seen, type-2 AGNs show no significant correlation (Spearman rank correlation coefficient, $r=0.16$, with a single-tailed $p=0.18$ ) between column density and reddening of their NLRs. Several things can be noted:

(i) The data do not support the claimed inverse correla-

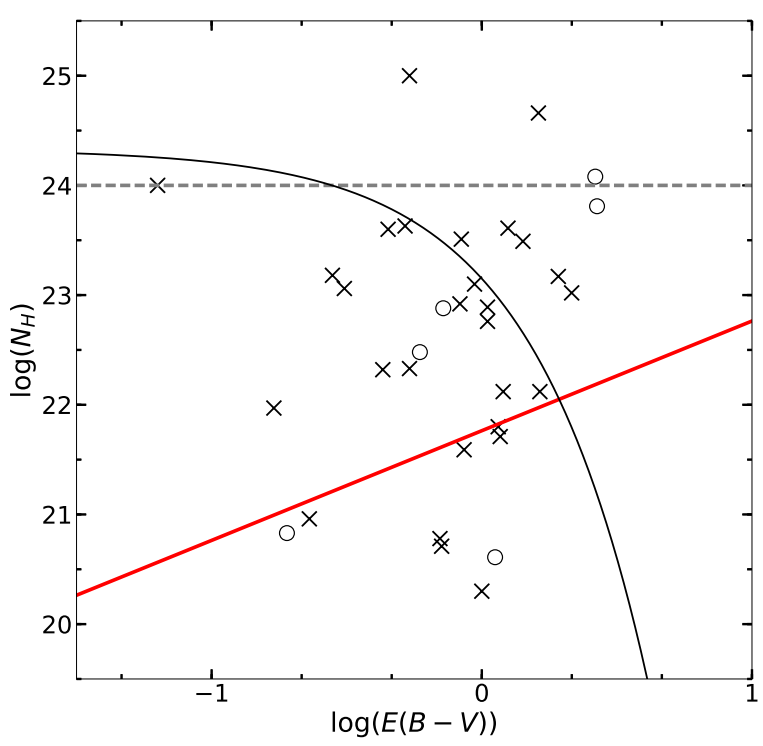

Figure 3. X-ray column densities versus NLR $\log E(B-V)$ for 35 type-2 AGNs and LINERs. Type-2 AGNs are shown as crosses and LINERs as open circles. The thick red line indicates the expected relationship between column density and reddening from Bohlin et al. (1978). The black curve shows the correlation claimed previously by Guainazzi et al. (2001). The dashed grey line indicates where an AGN becomes Compton-thick.

tion of Guainazzi et al. (2001) (shown by the curved line in Figure 3).

(ii) The column density is higher than predicted from the NLR by the Bohlin et al. (1978) relationship in the majority of cases.

(iii) The data set does not include a large number of Compton-thick AGNs.

\section{DISCUSSION}

\subsection{No dependence of Seyfert 2 column densities on NLR reddening}

The lack of correlation between $N_{H}$ and $E(B-V)$ for type-2 AGNs is not unexpected since the NLR gas is not usually on our direct line-of-sight to the black hole in type- 1 objects (see Fischer et al. 2013). There would only be a correlation if there were a common obscuring dusty screen covering both the NLR and the inner regions.

The higher X-ray columns in type-2 AGNs than those predicted from the NLR reddenings are consistent with the standard model where the obscuration of type-2 AGNs is withing the NLR.

There is no support for the curious anti-correlation claimed by Guainazzi et al. (2001) between $E(B-V)$ and $N_{H}$ for Seyfert 2 s. Such an anti-correlation would be hard to understand physically since it would imply that there was more dust when the total column density of gas was lower. 
The anti-correlation they reported is probably an artifact of excluding very high $N_{H}$ values.

\subsection{Column-density variability as the cause of the scatter in type-1 AGNs}

Although, as discussed above, there is a modest correlation between $E(B-V)$ and $N_{H}$ for Seyfert 1s, there a large scatter. We find this to be \pm 1.4 dex. Malizia et al. (1997) find significant variations in $N_{H}$ for most AGNs with repeated observations. Based on their reported variability given in their Table 1, we calculate that the scatter in $\log N_{H}$ due to variability and measuring error for a typical observation of an $\mathrm{AGN}$ is \pm 0.9 dex.

In addition to the effects of variability and measuring errors on $N_{H}$, there will also be errors in estimating $E(B-$ $V) . \mathrm{H} \alpha$ and $\mathrm{H} \beta$ fluxes are typically measured to an accuracy of $\pm 15 \%$. Thus the error in $\log E(B-V)$ will be about 0.40 dex.

Adding these effects in quadrature predicts a scatter of \pm 1.0 . Thus all of the scatter in Figure 1 could be due to column density variability and measuring errors if the reddening of the emission lines and continuum does not vary with changes in the hydrogen column along our line of sight to the X-ray emitting region. This is reasonable since partial coverage of the X-ray-emitting inner regions of AGNs (Reichert et al. 1985) implies that the size of absorbing clouds is comparable in size to the X-rayemitting region (less than a light-day in a typical Seyfert considered here). Variability of broad emission line profiles (Gaskell \& Harrington 2018) implies that dust clouds obscuring the BLR can often have sizes smaller than the typical $B L R$ radii of a few light weeks. Whilst there can be changes in reddening of the BLR, they will be less than changes in $N_{H}$ because a large area is being averaged. They will also generally not occur at the same time.

AGNs can show substantial changes in their Balmer line profiles. Also their Balmer decrements show changes both with velocity (i.e., across line profiles) and with time (see Gaskell \& Harrington 2018 and references therein). Gaskell \& Harrington (2018) have demonstrated that these changes, and puzzling changes in the time delays in response to continuum variability, can be explained by small dusty clouds partially obscuring the BLR. They propose that these clouds are part of the bi-conical outflow of dust in AGNs.

The timescale of variability of X-rays from the inner corona of the accretion disc shows that the X-ray-emitting region is much smaller than the lower-ionization BLR producing the Balmer lines. If the scenario of partial obscuration of the BLR is correct, then the small compact clouds must also at times cover the inner regions producing most of the X-rays. Lamer et al. (2003) show a good example of the variation in $N_{H}$ caused by a compact cloud passing front of the inner region of NGC 3227. Small-scale structure in absorbing clouds will introduce a lot of scatter in $N_{H}$ in Figures $1 \& 2$. Our sample in Table 1 includes galaxies with high column densities but low reddenings. These can be explained by having a compact obscuring cloud covering the X-ray corona, but mostly leaving the outer BLR unobscured. Conversely, when the BLR is reddened, but the X-rays are unobscured, we would be seeing the innermost regions through a hole in the obscuring material.

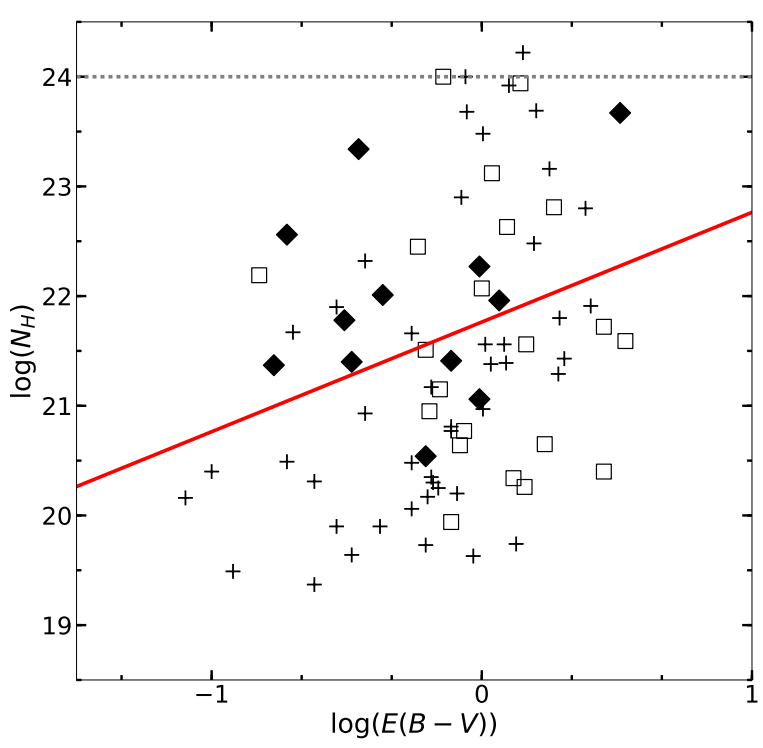

Figure 4. As for Figure 1 except that changing-look AGNs are shown as solid black diamonds and upper limit reddenings are not included.

\section{3 $\quad N_{H}$ predicted from $E(B-V)$}

In Figure 3, $N_{H}$ in type-2 AGNs is systematically greater than predicted by $E(B-V)$. This is unsurprising since there is much more central obscuration in type-2 AGNs and the line ratio is only giving the ratio from the NLR. However, for type-1 to type-1.9 AGNs (see Figures 1 and 2) there is no significant difference between the average observed and average predicted values if one uses the Galactic dust-to-gas ratio. The gas along our line of sight, much of it presumably expelled by the AGN, is therefore dusty.

\subsection{Scattered light?}

In type-2 AGNs there can be a substantial contribution of scattered light in X-ray spectra. This will dilute the photoelectric edge due to the absorbed X-rays and hence lead to lower column density estimates. We suggest that this is also going on to varying degrees in type $1-1.9$ AGNs. The effect will be to have moved points systematically to the bottom of Figure 1. Because of the difficulties of estimating $N_{H}$ it would be interesting to compare high-quality spectra of type-1 AGNs with both high and low reddenings to try to understand the differences. We suggest that a similar problem can arise in the optical. When the extinction is substantial even a small addition of scattered light to the optical spectra will change the observed Balmer decrement.

\section{5 "Changing-look" AGNs}

As noted above, large changes in $N_{H}$ are common in type1 and intermediate AGNs. There are also some AGNs that show substantial changes in their optical Seyfert type (see 
Oknyansky et al. 2017). These are known as "changing-look" AGNs (CL AGNs). The optical changes are in the strength of the broad components of the Balmer lines and in the continuum shape. The number of CL AGNs known at present is small, but many of them are well-know AGNs that have long been well-studied. This suggests that the real fraction of CL AGNs is quite high and that, as monitoring continues, many more AGNs will be classified as CL AGNs.

In Figure 4 we show the $N_{H}$ and $E(B-V)$ values for known optical CL AGNs compared with other AGNs that have not (yet) been seen to change their types. From this we see that, with the present sample, there are no obvious major differences between the CL AGNs and the AGNs not seen to have changed their types. Whilst the median values of both $N_{H}$ and $E(B-V)$ are higher for type 1.8 1.9 AGNs compared with type 1 - 1.5 AGNs (see Table 2), there is no statistically significant difference with the current small sample in these values for the CL AGNs. The $N_{H}$ and $E(B-V)$ values in the literature thus do not at present reveal any striking differences from non-CL AGNs. This is perhaps unsurprising since, by definition, CL AGNs are changing between types $1.8-1.9$ and types $1.0-1.5$. The lack of a significant difference is consistent with the idea that whatever is causing changing-looks, it is not due to fundamentally different amounts of obscuring material in CL AGNs.

\subsection{Compton-thick type 1s?}

In the simplest unified models (such as the "straw-person model" of Antonucci 1993), Seyfert 1 galaxies should not be Compton-thick. Alleged type-1 AGNs with claimed high column densities were therefore carefully scrutinized. We checked both whether the column density estimates are correct and whether the AGNs are really type-1s.

\subsubsection{Incorrect column densities?}

We examined the X-ray spectra of the three Seyfert 1s with published X-ray column densities around $10^{24} \mathrm{~cm}^{-2}$. After re-examining the X-ray spectra of these galaxies, NGC 3982, NGC 7674 (also known as Mrk 533), and NGC 6552, we found that, contrary to previous claims, they are not Compton-thick. The problem in all three cases is that the sources are weak, the X-ray spectra are noisy, and the background subtraction is uncertain. Two of the three show negative counts at low energies, thus indicating that too much background has been subtracted. The third shows a very strange spectrum. After adjustment of the background, probable absorption edges are seen in the two with negative spectra. Thus these AGNs cannot be Compton-thick because it is impossible for a Compton-thick object to show a strong absorption edge on its X-ray spectrum. Another object, NGC 1365, has a spectrum that shows a complex shape that is hard to interpret (see Risaliti et al. 2009). It would be beneficial to check more of the spectra to verify the column densities.

\subsubsection{Are the classifications correct?}

Optical spectra of allegedly type-1 AGNs with very high column densities were examined to check the classification. In all cases, these AGNs were found to be type-2s or at best type-1.9s. Details are as follows:

Even though some would classify NGC 424 as a Seyfert 1, the spectrum of Fosbury \& Sansom (1983) clearly shows that it is a Seyfert 2 (see their Figure 1). IC 5063, although classified as a Seyfert 1, can be clearly seen as a Seyfert 2 by the spectrum of Caldwell \& Phillips (1981). NGC 7582, although classified as a Seyfert 1, can be seen as a Seyfert 2 by the spectrum of Ward et al. (1978) Figure 5a. NGC 4507 and 3C 445 are seen as Seyfert 1s but are classified as Seyfert 1.9 s by Véron et al. (1981). NGC 1386 is misclassified as Seyfert 1, and is better classified as a Seyfert 2 by Terashima et al. (2002).

We thus conclude from the re-examination of X-ray and optical spectra that so far there is no evidence for any type-1 AGNs being Compton-thick.

\subsection{Unobscured Type 2s?}

Among the Seyfert 2 data are some Seyfert 2s that show a dramatically low column density for the proposed models. Much of this could be because of failure to recognize the effects of scattered light, but, as just discussed for type-1 AGNs, there can also be an issue with classification. The inclusion of non-thermal AGNs among the Seyfert 2 sample could give misleading results since non-thermal AGNs are fundamentally different (see Antonucci 2012) and might not have the surrounding dust. We found that a number of AGNs classified as type-2 in the literature were classified elsewhere as LINERs. These include, for example, NGC 4594 and NGC 5005 (Terashima et al. 2002) as well as six other AGNs.

\section{CONCLUSIONS}

We have made a compilation of reported estimates of the hydrogen column density, $N_{H}$, from X-ray spectra, and have made reddening estimates from broad and narrow Balmer decrements. The median BLR reddening is $E(B-V) \approx$ $0.77 \pm 0.10$ for type- 1 to type-1.9 AGNs with measured X-ray column densities. This is substantially greater than the the median reddening for AGNs found in the SDSS because the latter are biased towards blue AGNs by the SDSS selection criteria. We also find evidence of a publication bias against reporting low column densities.

We find that the observations of type- 1 to type1.9 AGNs are consistent with them following the standard Galactic relationship between $E(B-V)$ and $N_{H}$ of Bohlin et al. (1978). There is a large scatter of \pm 1.4 dex (without accounting for uncertainties in measurements) but the correlation is statistically significant. The Spearman onetailed probability for the null hypothesis of no correlation is $p=1.4 \times 10^{-5}$.

We find that known variability of $N_{H}$ is the dominant factor causing the scatter in the relationship between $E(B-V)$ and $N_{H}$ and that this plus observational errors in estimating $E(B-V)$ can fully account for the scatter.

We find that all previously reported claims Seyfert 1s 
with high column densities are spurious either because of misclassification of the optical spectra or low-quality X-ray spectra. We find no evidence for any cases of Compton-thick Seyfert 1s.

We find no significant correlation between column density and reddening of the NLR in type-2 AGNs. The puzzling anti-correlation claimed by Guainazzi et al. (2001) between the reddening of the NLR and $N_{H}$ in type-2 AGNs is not found

The median column density of LINERs is $22.68 \pm 0.75$ compared with $22.90 \pm 0.28$ for type-2 AGNs. We find that a number of reported Seyfert 2s with low column densities are LINERs, but $N_{H}$ is probably being underestimated in genuine type-2 AGNs because of scattered X-ray light diluting the photoelectric edge.

\section{ACKNOWLEDGMENTS}

We are grateful to Jane Turner, Ski Antonucci, and an anonymous referee for helpful comments on the paper.

\section{REFERENCES}

Abell, G. O., Eastmond, T. S., Jenner, D. C. 1978, ApJ, 221, L1 Alam, S., et al. 2015, ApJS, 219, 12

Antonucci, R. R. J. 1993, ARA\&A, 31, 473

Antonucci, R. R. J. 2012, Astron. \& Astrophys. Trans., 27, 557

Awaki, H., Koyama, K. 1993, ASR, 13, 221

Bolokovï̈̈Ğ, M., et al. 2014, ApJ, 794, 111

Barcons, X., Carrera, F. J., Ceballos, M. T. 2003, MNRAS, 346, 897

Bauer, F. E., et al. 2015, ApJ, 812, 116

Bianchi, S., Guainazzi, M., Matt, G., Chiaberge, M., Iwasawa, K., Fiore, F., Maiolino, R. 2005a, A\&A, 442, 185

Bianchi, S., Miniutti, G., Fabian, A. C., Iwasawa, K. 2005b, MNRAS, 360, 380

Bohlin, R. C., Savage, B. D., Drake, J. F. 1978, ApJ, 224, 132

Boller, T., Brandt, W. N., Fink, H. 1996, A\&A, 305, 53

Brinkmann, W., Siebert, J. 1994, A\&A, 285, 812

Burtscher L., et al., 2016, A\&A, 586, A28

Buttiglione, S., Capetti, A., Celotti, A, Axon, D. J., Chiaberge, M., Macchetto, F. D., Sparks, W. B. 2009, A\&A, 495, 1033

Cladwell, N., Phillips, M. M. 1981, ApJ, 244, 447

Canizares, C. R., McClintock, J. E., Ricker, G. R. 1978, ApJ, 226, L1

Cappi, M., et al. 2006, A\&A, 446, 459

Cohen, R. D. 1983, ApJ, 273, 489

Crenshaw, D. M., Kraemer, S. B. 2012, ApJ, 735, 75

Darhari, O., De Robertis, M. M. 1988, ApJS, 67, 249

de Bruyn, A. G., Sargent, W. L. W. 1978, AJ, 83, 1257

de Grijp, M. H. K., Keel, W. C., Miley, G. K., Goudfrooij, P., Lub, J. 1992, A\&AS, 96, 389

Denisyuk, É. K., Lipovetskii, V. A. 1977, PisâĂŹrna Astron. Zh., 3,7

Dong, X., Wang, T., Wang, J., et al. 2008, MNRAS, 383, 581

Edmunds, M. G., Pagel, B. E. J 1982, MNRAS, 198, 1089

Eracleous, M., Hwang, J. A., Flohic, H. M. L. G. 2010, MNRAS, 187,135

Falco, E. E., et al. 1999, PASP, 111, 438

Fischer, T. C., Crenshaw, D. M., Kraemer, S. B., Schmitt, H. R 2013, ApJ Supp., 209, 1

Fosbury, R. A. E., Sansom, A. E. 1983, MNRAS, 204, 1231

Fukazawa, Y., Iyomoto, N., Kubota, A., Matsumoto, Y., Makishima, K. 2001, A\&A, 374, 73
Gallo, L. C., Lehmann, I., Pietsch, W., Boller, Th., Brinkmann, W., Friedrich, P., Grupe, D. 2006, MNRAS, 365, 688

Gaskell, C. M. 1984, Astrophysical Letters, 24, 43

Gaskell, C. M. 2017, MNRAS, 467, 226

Gaskell C. M., Harrington P. Z., 2018, MNRAS, 478, 1660

George, I. M., Turner, T. J., Netzer, H. 1995, ApJ, 438, L67

Gilli, R. Vignali, C., Mignoli, M., Iwasawa, K., Comastri, A., Zamorani, G. 2010, A\&A, 519, A92

Glass, I. S. 1981, MNRAS, 197, 1067

Glass, I. S., Moorwood, A. F. M., Eichendorf, W. 1982, A\&A, 107,276

Gofford, J., Reeves, J. N., Tombesi, F., Braito, V., Turner, T. J., Miller, L., Cappi, M. 2013, MNRAS, 430, 60

González-Martín, O. 2008, Ph.D. Thesis, University of Granada

Goodrich R. W., Veilleux S., Hill G. J., 1994, ApJ, 422, 521

Grandi, S. A. 1981, ApJ, 251, 451

Guainazzi, M., Fiore, F., Matt, G., Perola, G. C., MNRAS, 327, 323

Guainazzi, M., Matt, G., Fiore, F., Perola, G. C. 2002, A\&A, 388, 787

Guainazzi, M., Rodriguez-Pascual, P., Fabian, A. C., Iwasawa, K., Matt, G. 2004, MNRAS, 355, 297

Guainazzi, M., Fabian, A. C., Iwasawa, K., Matt, G., Fiore, F. 2005, MNRAS, 356, 295

Hawley, S. A., Phillips, M. M. 1978, ApJ, 225, 780

Heard, C. Z. P., Gaskell, C. M. 2016, MNRAS, 461, 4227

Ho L. C., 2008, ARA\&A, 46, 475

Ho, L., C., Filippenko, A. V., Sargent, W. L. W. 1995, ApJS, 98, 477

Imanishi, M., Terashima, Y. 2004, AJ, 127, 758

Immler, S., et al. 2003, AJ, 126, 153

Isobe, T., Fiegelson, E. D., Akritas, M. G., Babu, G. J. 1990, ApJ, 364, 104

Jiménez-Bailón, E., Guainazzi, M., Matt, G., Bianchi, S., Krongold, Y., Piconcelli, E., Santos Lleó, M., Schartel, N. 2008, Rev. Mex. Astron. Astrofis., 32, 131

Jones, D. H., et al. 2009, MNRAS, 399, 683

Junkes, N., Zinnecker, H., Hensler, G., Dahlem, M., Pietsch, W. 1995, A\&A, 294, 8

Kartje, J. F., Königl, A., Hwang, C. Y., Bowyer, S. 1997, ApJ, 474,630

Khachikian, E. Y., Weedman, D. W. 1971, ApJ, 3, 389

Kollatschny, W., Schleicher, H., Fricke, K. J., Yorke, H. W. 1981, A\&A, 104, 198

Kunth, D., Sargent, W. L. W. 1979, A\&A, 76, 50

Lacy, J. H., et al. 1982, ApJ, 256, 75

Lamer, G., Uttley, P., McHardy, I. M. 2003, MNRAS, 342, L41

MacAlpine, G. M., Williams, G. A., Lewis, D. W. 1979, PASP, 97,746

Madejski, G. M., et al. 1995, ApJ, 438, 672

Makishima, K., et al. 1994, PASJ, 46, L77

Malizia, A., Bassani, L., Stephen, J. B., Malaguti, G., Palumbo, G. G. C. 1997, ApJS, 113, 311

Margon, B., Kwitter, K. B. 1978, ApJ, 224, L43

Marinucci, A., et al. 2014, MNRAS, 440, 2347

Markarian, B. E. 1977, A\&A, 58, 139

Markowitz, A., Reeves, J. N., George, I. M., Braito, V., Smith, R., Vaughan, S., Arévalo, P., Tombesi, F. 2009, ApJ, 691, 922

Marshall, F. E., et al. 1993, ApJ, 405, 168

Matt, G., Bianchi, S., D'Ammando, F., Martocchia, A. 2004, A\&A, 421, 473

Matt, G., Bianchi, S., Marinucci, A., Guainazzi, M., Iwawasa, K., Jiménez-Bailón, E. 2013, A\&A, 556, A91

McAlary, C. W., McLaren, R. A., Crabtree, D. R. 1979, ApJ, 234, 471

Mehdipour, M., et al. 2015, A\&A, 575, A22

Mihara, T., Matsuoka, M., Mushotzky, R. F., Kunieda, H., Otani, C., Miyamoto, S., Yamauchi, M. 1994, PASJ, 46, L137 
Miniutti, G., Piconcelli, E., Bianchi, S., Vignali, C., Bozzo, E. 2010, MNRAS, 401, 1315

Morris, S. L., Ward, M. J. 1988, MNRAS, 230, 639

Morse, J. A., Wilson, A. S., Elvis, M., Weaver, K. A. 1995, ApJ, 439,121

Moustakas, J., Kennicut Jr., R. C. 2006, ApJ Supp., 164, 81

Mulchaey, J. S., Colbert, E., Wilson, A. S., Mushotzky, R. F., Weaver, K. A. 1993, ApJ, 414, 144

Mulchaey, J. S., Koratkar, A., Ward, M. J., Wilson, A. S., Whittle, M., Antonucci, R. R. J., Kinney, A. L., Hurt, T. 1994, ApJ, 436,586

Mushotzky, R. F. 1982, ApJ, 256, 92

Nandra, K., Pounds, K. A. 1994, MNRAS, 268, 405

Nardini, E., et al. 2015, Science, 347, 860

Neugebauer, G., Oke, J. B., Becklin, E. E., Matthews, K. 1979, ApJ, 230, 79

Oknyansky, V. L., et al. 2017, MNRAS, 467, 1496

Osmer, P. S., Smith, M. G., Weedman, D. W. 1974, ApJ, 189, 187

Osterbrock, D. E., Koski, A. T., Phillips, M. M. 1976, ApJ, 206, 898

Osterbrock, D. E. 1977, ApJ, 215, 733

Osterbrock, D. E., \& Ferland, G. J. 2006, Astrophysics of gaseous nebulae and active galactic nuclei, 2nd. ed.

Osterbrock, D. E., Martel, A. 1993, ApJ, 414, 552

Petre, R., Mushotzky, R. F., Serlemitsos, P. J., Jahoda, K., Marshall, F. E. 1993, ApJ, 418, 644

Pfefferkorn, F., Boller, T., Rafanelli, P. 2001, A\&A, 368, 797

Phillips, M. M. 1978, ApJS, 38, 187

Phillips, M. M., Charles, P. A., Baldwin, J. A. 1983, ApJ, 266, 485

Piconcelli, E., Jiménez-Bailón, E., Guainazzi, M., Schartel, N., Rodríguez-Pascual, P. M., Santos-Lleó, M. 2004, MNRAS, 351, 161

Pietsh, W., Vogler, A., Kahabka, P., Jain, A., Klein, U. 1994, A\&A, 284, 386

Pineda, F. J., Delvaille, J. P., Grindlay, J. E., Schnopper, H. W. 1980, ApJ, 237, 414

Prieto, M. A., Pérez García, A. M., Rodríguez Espinosa, J. M. 2002, MNRAS, 329, 309

Puccetti, S., et al. 2014, ApJ, 739, 26

Puccetti, S., et al. 2016, A\&A, 585, A157

Rachen, J. P., Mannheim, K., Biermann, P. L. 1996, A\&A, 310, 371

Rebecchi, S., Bassani, L., Caroli, E., Di Cocco, G. 1992, Il Nuovo Cimento, 15, 889

Reichert, G. A., Mushotzky, R. F., Petre, R., \& Holt, S. S. 1985, ApJ, 296, 69

Rhee, G., Burns, J. O., Kowalski, M. P. 1994, AJ, 108, 1137

Ricci, C., et al. 2017, ApJS, 133, 17

Rieke, G. H. 1978, ApJ, 226, 550

Risaliti, G., Gilli, R., Maiolino, R., Salvati, M. 2000, A\&A, 357, 13

Risaliti, G., Elvis, M., Nicastro, F. 2002, ApJ, 571, 234

Risaliti, G., Elvis, M., Fabbiano, G., Baldi, A., Zezas, A. 2005, ApJ, 623, L93

Risaliti, G., et al. 2009, ApJ, 705, L1

Rivers, E., Markowitz, A., Duro, R., Rothschild, R. 2012, ApJ, 759,63

Rivers, E., et al. 2015, ApJ, 815, 55

Rosales-Ortega, F. F., et al. 2010, MNRAS, 405, 735

Różańska, A., Czerny, B., Siemiginowska, A., Dumont, A. -M., Kawaguchi, T. 2004, ApJ, 600, 96

Rudy, R. J. 1984, ApJ, 284, 33

Ruschel-Dutra, D., Pastoriza, M., Riffel, R., Sales, D. A., Winge, C. 2014, MNRAS, 438, 3434

Saez, C., Brandt, W. N., Gallagher, S. C., Bauer, F. E., Garmire, G. P. 2012, ApJ, 759, 42
Schartel, N., et al. 1996, MNRAS, 283, 1015

J. L., Antonucci, R. R. J., et al. 2003, ApJ, 597, 768

Shi Y., et al., 2006, ApJ, 653, 127

Shu, X. W., Wang, J. X., Jiang, P., Fan, L. L., Wang, T. G. 2007, ApJ, 657, 167

Shu, X. W., Yaqoob, T., Murphy, K. D., Braito, V., Wang, J. X., Zheng, W. 2010, ApJ, 713, 1256

Smith, D. A., Done, C. 1996, MNRAS, 280, 355

Storchi-Bergmann, T., Kinney, A. L., Challis, P. 1995, ApJS, 98, 103

Sun, S., Guainnazi, M., Ni, Q., Wang, J., Qian, Shi, F., Wang, Y., Bambi, C. 2016, arXiv:1704.03716v1

Terashima, Y., Iyomoto, N., Ho, L. C., Ptak, A. F. 2002, ApJ, 139,1

Tohline, J. E., Osterbrock, D. E. 1976, ApJ, 210, L117

Torrealba, J., Chavushyan, V., Cruz-González, I., Arshakian, T. G., Bertone, E., Rosa-González, D. 2012, Rev. Mex. Astron. Astrofis., 48, 9

Tueller, J., Mushotzky, R. F., Barthelmy, S., Cannizzo, J. K., Gehrels, N., Marhwardt, C. B., Skinner, G. K., Winter, L. M. 2008, ApJ, 681, 113

Turner, T. J., Pounds, K. A. 1989, MNRAS, 240, 833

Turner, T. J., Nandra, K., George, I. M., Fabian, A. C., Pounds, K. A. 1993, ApJ, 419, 127

Turner, T. J., Miller, L., Kraemer, S. B., Reeves, J. N. 2011, ApJ, 733,48

Ueno, S., Mushotzky, R. F., Koyama, K., Iwasawa, K., Awaki, H., Hayashi, I. 1994, PASJ, 46, L71

Ursini, F., et al. 2015, MNRAS, 452, 3266

Vasudevan, R. V., Brandt, W. N., Mushotzky, R. F., Winter, L. M., Baumgartner, W. H., Shimizu, T. T., Schneider, D. P., Nousek, J. 2013, ApJ, 763, 111

Véron, P., Véron, M. P., Zuiderwijk, E. J. 1981, A\&A, 102, 116

Véron-Cetty, M. -P., Véron, P. 1986, A\&AS, 66, 335

Veilleux S., Goodrich R. W., Hill G. J., 1997, ApJ, 477, 631

Walter, R., Fink, H. H. 1993, A\&A, 274, 105

Wang, T., Brinkmann, W., Bergeron, J. 1996, A\&A, 309, 81

Wang, J., Zhang, J., Fan, J. 2010, Res. Astron. Astrophys., 10, 915

Ward, M. J., Wilson, A. S., Penston, M. V., Elvis, M., Maccacaro, T., Tritton, K. P. 1978, ApJ, 223, 788

Ward, M., Allen, D. A., Wilson, A. S., Smith, M. G., Wright, A. E. 1982, MNRAS, 199, 953

Warwick, R. S., Sembay, S., Yaqoob, T., Makishima, K., Ohashi, T., Tashiro, M., Kohmura, Y. 1993, MNRAS, 265, 412

Weaver, K. A., Yaqoob, T., Holt, S. S., Mushotzky, R. F., Matsuoka, M., Yamauchi, M. 1994, ApJ, 436, L27

Weaver, K. A., Arnaud, K. A., Mushotzky, R. F. 1995, ApJ, 447, 121

Weaver, K. A., Nousek, J., Yaqoob, T., Mushotzky, R. F., Makino, F., Otani, C. 1996, ApJ, 458, 160

Weingartner, J. C., Draine, B. T. 2001, ApJ, 548, 296

White, N. E., Giommi, P., Angelini, L. 1994, Bulletin of the American Astronomical Society, 26, 41.11

Wilson, A. S., Penston, M. V. 1979, ApJ, 232, 389

Winter, L. M., Mushotzky, R. F., Tueller, J., Markwardt, C. 2008, ApJ, 674, 686

Winter, L. M., Mushotzky, R. F., Reynolds, C. S., Tueller, J. 2009, ApJ, 690, 1322

Winter, L. M., Lewis, K. T., Koss, M., Veilleux, S., Keeney, B., Mushotzky, R. F. 2010, ApJ, 710, 503

Wysota, A., \& Gaskell, C. M. 1988, Active Galactic Nuclei, 307, 79

Yamada, S., Ueda, Y., Oda, S., Tanimoto, A., Imanishi, M., Terashima, Y., Ricci, C. 2018, ApJ, 858, 106

Ying, T., JunXian, W., Kai, Z. 2012, Science China, 55, 2482

Zhang, J. S., Henkel, C., Kadler, M., Greenhill, L. J., Nagar, N., Wilson, A. S., Braatz, J. A. 2006, A\&A, 450, 93 


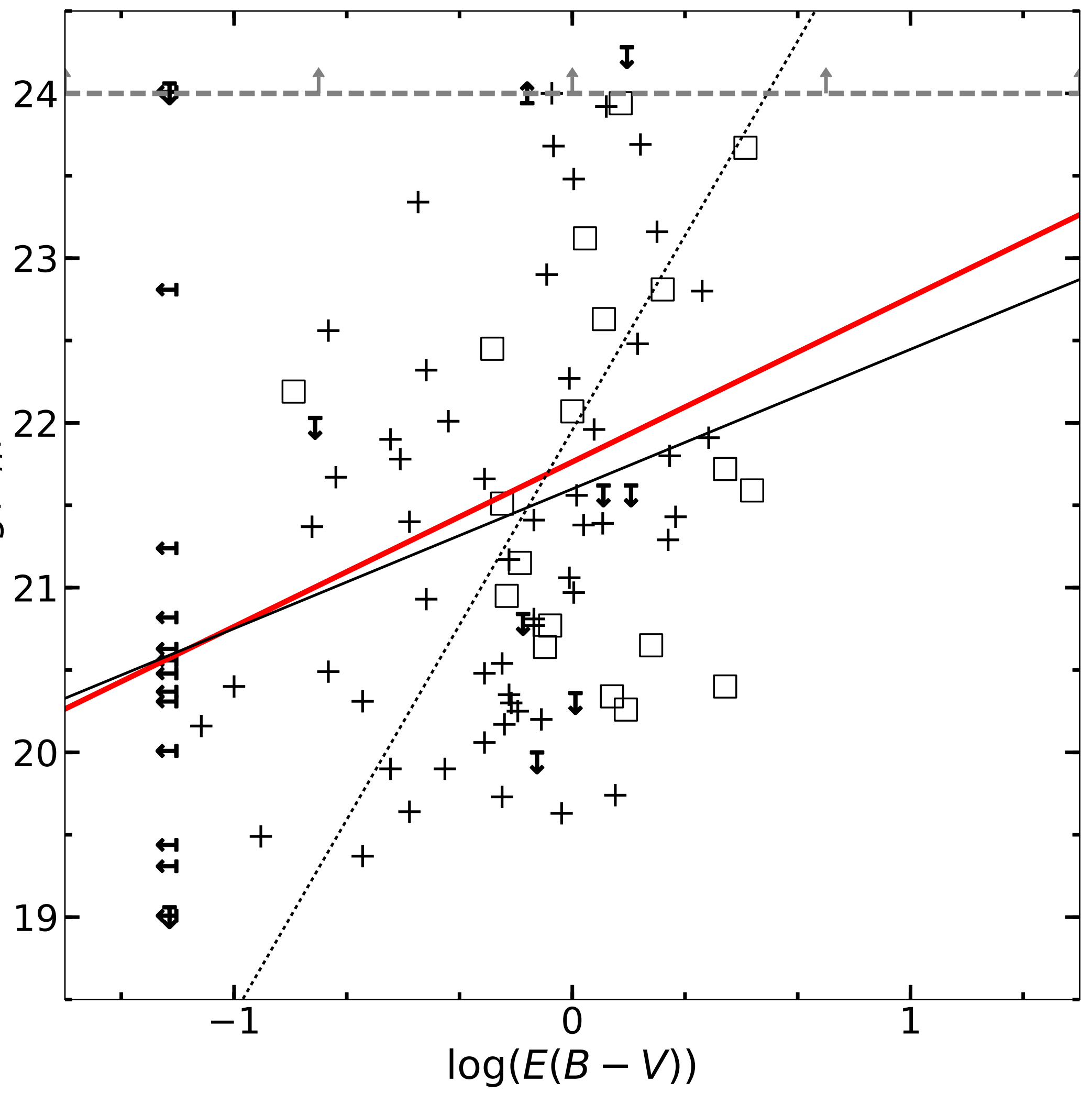

